precession (SSFP) cine images. FT-CMR analysis was performed offline using tissue-tracking software (CVI42, Circle Cardiovascular Imaging Inc.) to estimate LV global longitudinal strain (GLS) from two long-axis SSFP cine images (Figure 1). To correct for the LV remodelling process, LV GLS was corrected for LV end-diastolic volume.

Results There were significant differences in LV volumes, mass and ejection fraction across the 3 groups of patients (Table 1): patients with aortic regurgitation showed significantly larger LV volumes, and lower LVEF compared to patients with normal aortic valve function and patients with aortic stenosis. There were no differences in LV GLS across the groups. However, after correcting for LV end-diastolic volume, patients with aortic regurgitation showed more impaired LV GLS as compared to the other groups.

\begin{tabular}{|c|c|c|c|c|}
\hline & $\begin{array}{l}\text { Normal valve } \\
\text { function }(n=14)\end{array}$ & $\begin{array}{l}\text { Aortic stenosis } \\
(\mathrm{n}=29)\end{array}$ & $\begin{array}{l}\text { Aortic } \\
\text { regurgitation }(n= \\
\text { 28) }\end{array}$ & $\begin{array}{l}\text { ANOVA } \\
\text { p-value }\end{array}$ \\
\hline $\begin{array}{l}\text { Heart rate } \\
\text { (beats } / \mathrm{min} \text { ) }\end{array}$ & $71 \pm 8$ & $70 \pm 12$ & $64 \pm 13$ & 0.132 \\
\hline LVEDV (ml) & $135 \pm 33$ & $138 \pm 33$ & $186 \pm 50^{*}$ & $<0.001$ \\
\hline LVESV (ml) & $46 \pm 15$ & $43 \pm 16$ & $70 \pm 22^{*}$ & $<0.001$ \\
\hline LVEF (\%) & $65 \pm 5$ & $69 \pm 6$ & $62 \pm 5 \dagger$ & $<0.001$ \\
\hline LV mass (gr) & $120 \pm 29^{\#}$ & $153 \pm 36$ & $157 \pm 35$ & 0.005 \\
\hline GLS (\%) & $-19.2 \pm 3.4$ & $-19.3 \pm 3.6$ & $-19.8 \pm 3.0$ & 0.813 \\
\hline $\begin{array}{l}\text { LV GLS/ } \\
\text { LVEDV }\end{array}$ & $-0.15 \pm 0.05$ & $-0.14 \pm 0.04$ & $-0.12 \pm 0.04^{*}$ & 0.01 \\
\hline
\end{tabular}

${ }^{*} \mathrm{p}<0.001$ vs. Aortic stenosis and normal; $\dagger \mathrm{p}<0.001$ vs. Aortic stenosis; ${ }^{\#} \mathrm{p}<0.001$ vs. Aortic regurgitation and Aortic stenosis.

Conclusions LV mechanics significantly differ across normal functioning and different type of aortic valve dysfunction (stenosis and regurgitation), with aortic regurgitation showing the most impaired LV GLS corrected for LV end-diastolic volume, despite preserved LVEF.

\section{INTRA-VENTRICULAR MYOCARDIAL DEFORMATION STRAIN ANALYSIS IN HEALTHY VOLUNTEERS: REGIONAL VARIATION AND IMPLICATIONS FOR REGIONAL MYOCARDIAL DISEASE PROCESSES}

1,2,*A Scatteia, 'J Rodrigues, 'S Lyen, 'E De Gerate, ${ }^{1} \mathrm{~A}$ Baritussio, ${ }^{1} \mathrm{~A}$ Ghosh Dastidar ${ }^{1} \mathrm{G}$ Biglino, ${ }^{3} \mathrm{~A}$ Maceira, ${ }^{3} \mathrm{D}$ Pennell, ${ }^{1} \mathrm{C}$ Bucciarelli-Ducci. 'Bristol Heart Institute, University Hospitals Bristol NHS Foundation Trust, UK; ${ }^{2}$ Federico II, University of Naples, Naples, Italy; ${ }^{3}$ Brompton NIHR Cardiovascular Biomedical Research Unit, London, UK

10.1136/heartjnl-2016-309668.22

Ejection fraction (EF) is a traditional marker of systolic function. However, it may not detect early, subtle cardiac disease with regional predilection. The aim of this study was to define regional intra-ventricular variation in myocardial strain in a cohort of healthy volunteers using Tissue-tracking cardiac magnetic resonance (CMR).

Methods Healthy volunteers were recruited $(\mathrm{n}=94$, age range 20-79 years, 54\% male). CMR at $1.5 \mathrm{~T}$ was performed. Tissuetracking software (CVI42, Circle Cardiovascular Imaging Inc.) estimated myocardial strain from the long-axis and the short-axis steady-state free precession (SSFP) cine images (Figure 1). The entire cohort was analysed by two independent readers. Interobserver variability was also assessed. Myocardial segments were defined in accordance to the American Heart Association 16-segment model. Regional variations in circumferential and radial strain between basal, mid-cavity and apical segments as well as between left ventricle walls were assessed. Statistical analysis was performed using paired t test $(\mathrm{p}<0.05)$.

Results Inter-observer reproducibility analyses were excellent for mid-cavity and apical radial and circumferential strain values. On the other hand, reproducibility was not as good for basal segments for both deformation directions. Regional variations in strain (Table 1) revealed a statistically significant increase in deformation of the apical segments compared to the basal and mid-cavity ones for both radial and circumferential strain. Analysis of the different LV walls deformations indicated lowest values in the septum in all subjects, as well as across all age and gender subgroups.

Conclusion This is the first study to demonstrate that there is a positive gradient toward the apex in both circumferential and radial strain using CMR-derived myocardial strain analysis.

Abstract 22 Table 1 Regional strain values

\begin{tabular}{|c|c|c|c|c|c|c|c|c|c|c|c|}
\hline Strain $\%$ & Subgroup & & Basal & Mid-cavity & Apical & P-value* & Septal & Lateral & Anterior & Inferior & P-value** \\
\hline \multirow[t]{7}{*}{ Circumferential } & Gender & & & & & & & & & & \\
\hline & & Male $(n=51)$ & $-19 \pm 2$ & $-20 \pm 2$ & $-24 \pm 2$ & $<0.001$ & $-16 \pm 2$ & $-24 \pm 3$ & $-22 \pm 3$ & $-22 \pm 2$ & $<0.001$ \\
\hline & & Female $(n=43)$ & $-20 \pm 2$ & $-21 \pm 3$ & $-24 \pm 3$ & $<0.001$ & $-17 \pm 3$ & $-24 \pm 3$ & $-23 \pm 3$ & $-22 \pm 2$ & $<0.001$ \\
\hline & Age (years) & & & & & & & & & & \\
\hline & $20-39(n=28)$ & & $-19 \pm 2$ & $-19 \pm 2$ & $-23 \pm 3$ & $<0.001$ & $-15 \pm 3$ & $-23 \pm 3$ & $-22 \pm 3$ & $-21 \pm 2$ & $<0.001$ \\
\hline & $40-59(n=35)$ & & $-19 \pm 2$ & $-20 \pm 2$ & $-24 \pm 2$ & $<0.001$ & $-16 \pm 3$ & $-24 \pm 2$ & $-22 \pm 3$ & $-22 \pm 2$ & $<0.001$ \\
\hline & $60-79(n=31)$ & & $-20 \pm 2$ & $-22 \pm 2$ & $-25 \pm 3$ & $<0.001$ & $-17 \pm 2$ & $-26 \pm 2$ & $-24 \pm 2$ & $-23 \pm 2$ & $<0.001$ \\
\hline \multicolumn{12}{|l|}{ Radial } \\
\hline & Gender & & & & & & & & & & \\
\hline & & Male $(n=51)$ & $39 \pm 6$ & $37 \pm 7$ & $51 \pm 9$ & $<0.001$ & $28 \pm 6$ & $52 \pm 11$ & $46 \pm 9$ & $44 \pm 8$ & $<0.001$ \\
\hline & & Female $(n=43)$ & $43 \pm 8$ & $39 \pm 9$ & $52 \pm 11$ & $<0.001$ & $30 \pm 8$ & $52 \pm 11$ & $48 \pm 11$ & $45 \pm 8$ & $<0.001$ \\
\hline & Age (years) & & & & & & & & & & \\
\hline & & $20-39(n=28)$ & $39 \pm 7$ & $34 \pm 7$ & $48 \pm 10$ & $<0.001$ & $27 \pm 7$ & $47 \pm 9$ & $45 \pm 10$ & $42 \pm 6$ & $<0.001$ \\
\hline & & $40-59(n=35)$ & $40 \pm 6$ & $37 \pm 6$ & $52 \pm 10$ & $<0.001$ & $28 \pm 8$ & $50 \pm 9$ & $44 \pm 10$ & $42 \pm 9$ & $<0.001$ \\
\hline & & $60-79(n=31)$ & $44 \pm 9$ & $43 \pm 9$ & $55 \pm 11$ & $<0.001$ & $32 \pm 5$ & $60 \pm 11$ & $51 \pm 9$ & $49 \pm 8$ & $<0.001$ \\
\hline
\end{tabular}



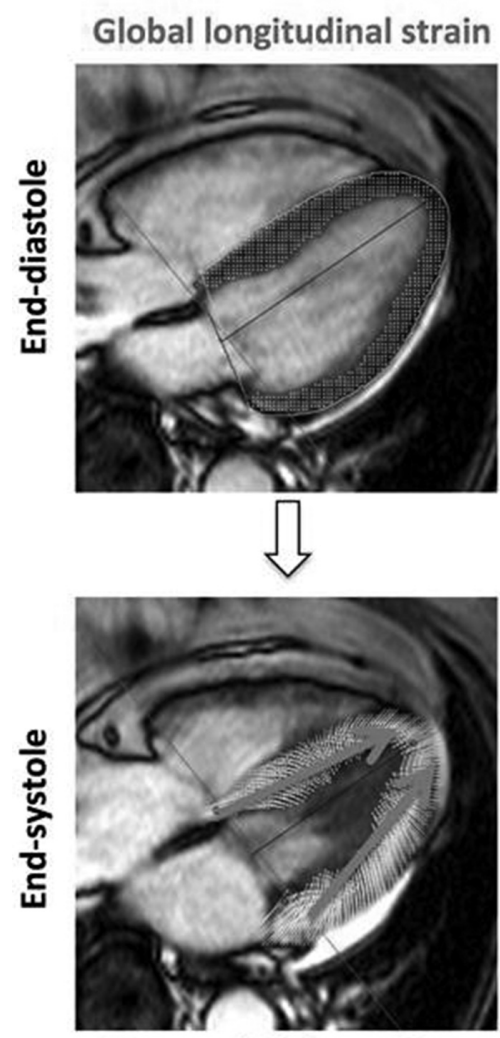

Myocardial shortening (conventionally-ve value)
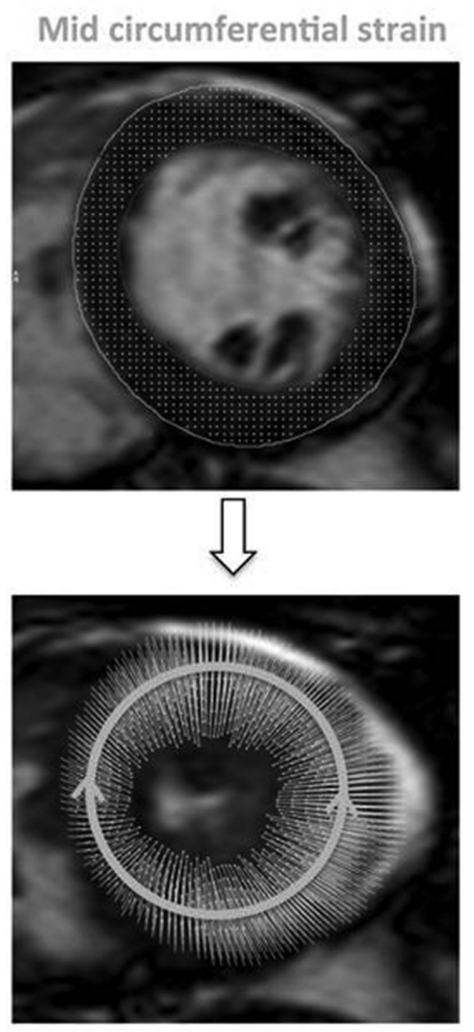

Myocardial shortening (conventionally-ve value)

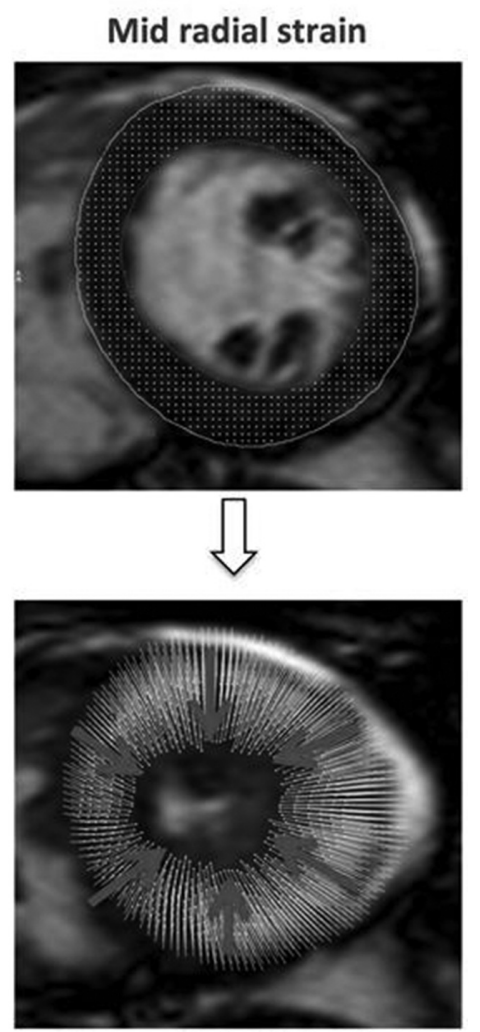

Myocardial thickening (conventionally +ve value)

Abstract 22 Figure 1 Definition of longitudinal, circumferential and radial myocardial strain, calculated by SSFP long-axis and short-axis cine images

Furthermore, we also showed that the interventricular septum is the segment with lowest deformation values. These findings are important, as a comprehensive understanding of normal intraventricular regional variation is needed before this new tool can be implemented in routine clinical practice.

\section{ASSESSMENT OF THE ISCHAEMIC BURDEN IN PATIENTS WITH ISCHAEMIC HEART DISEASE THROUGH COMBINED HIGH-RESOLUTION ASSESSMENT OF QUANTITATIVE PERFUSION AND LATE ENHANCEMENT}

*ADM Villa, E Sammut, JS Shome, S Plein, R Razavi, A Chiribiri. Division of Imaging Sciences and Biomedical Engineering, Department of Cardiovascular Imaging, King's College London, London, UK

\subsection{6/heartjnl-2016-309668.23}

Background Patients with ischaemic heart disease (IHD) often show a combination of inducible ischaemia and previous myocardial infarction, therefore is particularly relevant to reach an accurate assessment of myocardial viability and ischaemic burden, as this may results in more appropriate therapy and better outcome. Areas of scar frequently result in false-positive perfusion findings, we therefore hypothesised that combined cardiac magnetic resonance (CMR) high-resolution quantitative perfusion and late gadolinium enhancement (LGE) protocol will result in a more accurate evaluation of ischaemic burden, avoiding areas of scar.
Methods 15 patients with IHD and ejection fraction (EF) $<45 \%$ were included. Patients underwent adenosine stress perfusion at 3T (Philips Achieva) using high-resolution kt turbo-field-echo sequence and dual bolus approach. Perfusion and LGE images were analysed both qualitatively and quantitatively (using validated high-resolution deconvolution analysis and conventional semi-quantitative analysis with 5SD). For combined analysis, perfusion and LGE images were matched in terms of position and cardiac phase using a deformable template segmentation method. High-resolution MPR and LGE maps were then generated and ischaemic burden calculated \pm LGE (Figure 1).

Results The average EF was $33 \pm 9.5 \%$. All patients showed scar and perfusion defects at visual assessment. The average scarred area was $18 \pm 6.8 \%$. Average MPR was $2.3 \pm 2.4,3.2$ \pm 0.6 in viable area (LGE-) and $1.05 \pm 0.69$ in non-viable areas $(\mathrm{LGE}+)(\mathrm{p}=0.001$ Vs LGE-). $27 \%(4 / 15)$ of patients had a perfusion defect extending only in LGE area. The overall ischaemic burden (MPR threshold 1.5) was $23.2 \pm 13.5 \%$, but after excluding LGE dropped to $12.4 \pm 7.6 \%(\mathrm{p}=0.001)$.

Conclusions Our study demonstrates the potential of combined high-resolution assessment of stress perfusion and LGE to provide more accurate measurements of ischaemic burden excluding areas of scar, These areas, which frequently result in false positive perfusion defects and possible overestimation of ischaemic burden, had an $M P R \leq 1$, as expected in areas of scar. 